\title{
Características clínicas y complicaciones de la cirrosis hepática en una población de altura (Huancayo, 3250 m s. n. m.)
}

Calderón Gerstein, Walter*1,2,a; Ascanio Paredes, Maritza 1,b; Yarinsueca Mata, Pilar Rocío 3,c

\section{RESUMEN}

Objetivo: La cirrosis hepática ocasiona significativa mortalidad y morbilidad. Esta investigación trata de determinar la presentación clínica, la etiología y las complicaciones de los pacientes con cirrosis hepática en una población que habita en una región de altura del Perú.

Materiales y métodos: Evaluación retrospectiva de la presentación clínica y las complicaciones de la cirrosis hepática. Se estudiaron 108 historias clínicas de pacientes cirróticos ingresados en el Servicio de Medicina del Hospital Nacional Ramiro Prialé Prialé de la ciudad de Huancayo entre el 2010 y el 2012.

Resultados: El promedio de edad de los pacientes fue de 60,50 años (rango 12-82 años) y el 62,90\% fueron varones. La etiología más frecuente fue la ingesta alcohólica (63,00\%) seguida por las hepatitis B y C crónicas (7,40 \% y 2,80\%, respectivamente). El 58,30\% de los casos correspondía al estadio B de la clasificación de Child-Pugh y el 31,30\% se encontraba en estadio C. La manifestación clínica más frecuente fue la distensión abdominal $(87,00 \%)$. Las complicaciones más comunes fueron la ascitis (56,00 \%), la encefalopatía hepática $(47,20 \%)$ y el síndrome hepatorrenal $(8,30 \%)$.

Conclusiones: La cirrosis alcohólica fue la etiología más común y las complicaciones más frecuentes fueron la ascitis y la encefalopatía hepática.

Palabras clave: Cirrosis hepática; Ascitis; Encefalopatía hepática; Síndrome hepatorrenal (Fuente: DeCS BIREME).

\section{Clinical features and complications of liver cirrhosis in a high-altitude population (Huancayo, 3,250 m a.s.l.)}

\begin{abstract} region in Peru. Hospital Nacional Ramiro Prialé Prialé, Huancayo, were evaluated between 2010 and 2012. encephalopathy (47.2\%) and hepatorenal syndrome $(8.3 \%)$. hepatic encephalopathy.

Keywords: Liver cirrhosis; Ascites; Hepatic encephalopathy; Hepatorenal syndrome (Source: MeSH NLM).

\footnotetext{
1 Departamento de Medicina. Hospital Nacional Ramiro Prialé Prialé-Essalud. Huancayo, Perú.

2 Universidad Continental. Huancayo, Perú.

3 Hospital Luís Negreiros Vega. Essalud. Callao, Perú.

a Médico internista.

b Médico cardióloga.

c Médico pediatra.

* Autor corresponsal.
}

Objective: Liver cirrhosis is responsible for significant morbidity and mortality. This research aims to determine the clinical presentation, etiology and complications of patients with liver cirrhosis from a population living at a high altitude

Materials and methods: A retrospective study of the clinical presentation and complications of liver cirrhosis was conducted. One hundred eight (108) medical records of patients with liver cirrhosis admitted to the Internal Medicine Service of the

Results: Patients' mean age was 60.5 years (range: $12-82$ years) and $62.9 \%$ were males. The most common etiology was alcohol consumption (63\%), followed by chronic hepatitis B and C (7.4\% and $2.8 \%$, respectively). Fifty-eight point three percent (58.3\%) of the patients had a Child-Pugh class B score and $31.30 \%$ of them had a Child-Pugh class C score. The most frequent clinical presentation was abdominal distension (87\%). The most common complications were ascites (56\%), hepatic

Conclusions: Alcoholic liver cirrhosis was the most common etiology. The most frequent complications were ascites and 


\section{INTRODUCCIÓN}

La morbilidad y mortalidad de la cirrosis hepática es elevada, es la quinta o sexta causa general de fallecimiento en México ${ }^{(1)}$ y Chile ${ }^{(2)}$. En el Perú, la tasa de mortalidad de esta enfermedad es de 9,48 pacientes fallecidos por cien mil habitantes. Además, ocupa el quinto lugar en la mortalidad general, pero en la población de 20 a 64 años representa la segunda causa de muerte ${ }^{(3)}$. En Huancayo, Junín, la cirrosis es la tercera causa de mortalidad después de las neumonías y el cáncer de estómago, con una tasa de 11 casos por cada 100 mil habitantes, y se establece como el principal motivo de mortalidad en los adultos de 20 a 64 años ${ }^{(4)}$.

En el Perú, según Dávalos ${ }^{(5)}$ la cirrosis hepática sería más prevalente en varones mayores de 60 años. En una revisión de estudios epidemiológicos, el mencionado autor concluyó que el tipo principal sería la cirrosis alcohólica o de Laennec y la segunda, la hepatitis crónica por virus $B$. Los casos de cirrosis se diagnosticarían en un estado avanzado ${ }^{(5)}$, especialmente cuando aparece una complicación, entre las cuales, la hemorragia por várices esofágicas sangrantes sería la más frecuente.

En la altura, la presión de oxígeno y la temperatura son menores, lo que condiciones cambios en las manifestaciones de diversas enfermedades como las respiratorias, cardiovasculares y gastrointestinales ${ }^{(6,7)}$. En la ciudad de Huancayo, ubicada a 3259 m s. n. m., la población no solo está expuesta a los efectos de la altura, sino que presenta características socioculturales y económicas propias que podrían influir en la etiología y formas de presentación de cuadros digestivos como la cirrosis. Debido a que no existen estudios de esta enfermedad en poblaciones de altura por encima de los 3000 m s. n. m., por lo que se realizó una investigación con el fin de conocer la forma de presentación clínica, así como las complicaciones más frecuentes en los pacientes hospitalizados con cirrosis hepática.

Los resultados obtenidos permitirán conocer mejor el perfil clínico del paciente cirrótico en la altura y beneficiarán a la población andina del Perú en lo concerniente a la identificación y prevención de complicaciones y al diagnóstico precoz.

\section{MATERIALES Y MÉTODOS}

\section{Tipo y diseño de estudio}

La presente investigación es un estudio epidemiológico de tipo descriptivo, retrospectivo y transversal.

\section{Población de estudio}

Fueron evaluadas las historias clínicas de los pacientes admitidos al Servicio de Medicina del Hospital Nacional Ramiro Prialé Prialé de EsSalud de la ciudad de Huancayo (3259 m s. n. m.) en el periodo del 2010 al 2012.
Se revisaron 142 historias clínicas y se excluyeron 34 porque los datos estaban incompletos. Los datos clínicos y de laboratorio que se registraron en la investigación provenían de la información obtenida en las primeras 24 horas de hospitalización.

Los criterios de inclusión fueron los siguientes: pacientes con evidencia de hepatopatía crónica que cumplían con los ya mencionados criterios diagnósticos para cirrosis, y que habían vivido a una altura por encima de los 3000 m s. n. m. durante los últimos cinco años. Se excluyeron a los pacientes con evidencia de hepatopatía crónica pero que no cumplían con los criterios diagnósticos mencionados e individuos que no habían vivido a una altura por encima de los 3000 m s. n. m. durante los últimos cinco años.

\section{Variables y mediciones}

La cirrosis hepática fue diagnosticada por biopsia o por ciertas características clínicas, ecográficas y bioquímicas. Además, fue indispensable la evidencia tanto de insuficiencia hepatocelular como de hipertensión portal. La falla o insuficiencia hepatocelular fue documentada por la presencia de, como mínimo, de tres de las siguientes alteraciones: hipoalbuminemia (con otras causas descartadas), relación TGP/TGO invertida con valores disminuidos de TGO, encefalopatía hepática confirmada terapéuticamente, relación albúmina/globulina invertida, ginecomastia o pérdida del vello androgénico.

La hipertensión portal fue diagnosticada por una de las siguientes alteraciones: ascitis (de la variedad trasudado y sin otra etiología), confirmación de hipertensión en la vena porta mediante ecografía Doppler venosa, identificación de várices esofágicas por vía endoscópica, circulación abdominal colateral tipo caput Medusae y presencia de síndrome de Banti (trombocitopenia o leucopenia asociada a esplenomegalia). Como criterio diagnóstico complementario se consideró el hallazgo de lesiones micro o macronodulares compatibles con cirrosis en exploraciones hepáticas por laparoscopía, sonografía o tomografía.

Las complicaciones asociadas a cirrosis fueron las siguientes: encefalopatía hepática, peritonitis bacteriana espontánea, síndrome hepatorenal y sangrado por várices esofágicas.

\section{Análisis estadístico}

Las variables categóricas fueron analizadas con la prueba de Chi cuadrado y las continuas con t de Student. Se realizó el test de Kolmogorov-Smirnov (KS) para determinar la normalidad en el análisis univariado.

\section{Consideraciones éticas}

El estudio fue aprobado por los Comités de Ética y de Investigación del Hospital Nacional Ramiro Prialé Prialé EsSalud-Huancayo. 


\section{RESULTADOS}

Fueron evaluadas 108 historias clínicas correspondientes a igual número de pacientes. La distribución de la población fue normal para el test de KS. La mayoría de la población $(62,90 \%)$ era de sexo masculino y el $37,10 \%$ eran mujeres. La edad promedio fue de 60,50 años. Los individuos entre los 60 y 69 años constituyeron el grupo más numeroso (33,30\%), en segundo lugar, los que tenían entre 50 y 59 años $(25,00 \%)$ y, finalmente, el grupo entre 70 y 80 años ( $22,00 \%$ ).
Presentación clínica: El peso promedio de los pacientes fue $61,04 \mathrm{~kg}$ y la talla promedio fue 1,57 m. La media del índice de masa corporal (IMC) fue de $23,2 \mathrm{~kg} / \mathrm{m}^{2}$.

La lesión hepática alcohólica fue la causa más frecuente de cirrosis hepática $(63,00 \%, n=68)$, seguida de hepatitis B $(7,40 \%, n=8)$. Tres pacientes tuvieron hepatitis C; de ellos, uno presentó cirrosis biliar primaria y otro cirrosis alcohólica y hepatitis B. En el 25,00 \% ( $n=27)$ de los casos no se identificó etiología (Tabla 1).

Tabla 1. Etiología de la cirrosis hepática

\begin{tabular}{|ccc|}
\hline Etiología & Casos & $\%$ \\
\hline Cirrosis alcohólica & 68 & 63,00 \\
\hline Hepatitis B crónica & 8 & 7,40 \\
\hline Hepatitis C crónica & 3 & 2,80 \\
\hline Cirrosis biliar primaria & 1 & 0,90 \\
\hline Hepatitis B y alcohol & 1 & 0,90 \\
\hline Cirrosis criptogénica & 27 & 25,00 \\
\hline Total & 108 & 100,00 \\
\hline
\end{tabular}

En el examen físico, la distensión abdominal fue el signo más frecuente $(n=88 ; 81,40 \%)$. La identificación de matidez desplazable $(n=81,75 \%)$ y la confirmación ecográfica de ascitis ( $\mathrm{n}=77 ; 71,30 \%)$ también se observaron con frecuencia (Tabla 2).

Tabla 2. Hallazgos al examen físico

\begin{tabular}{|ccc|}
\hline Signos & Número & $\%$ \\
\hline Distensión abdominal & 87 & 81,30 \\
\hline Matidez desplazable & 81 & 75,00 \\
\hline Ascitis & 77 & 71,30 \\
\hline Ictericia & 64 & 59,30 \\
\hline Edemas & 49 & 45,40 \\
\hline Signo de la oleada & 42 & 38,90 \\
\hline Circulación colateral & 33 & 30,60 \\
\hline Equimosis & 27 & 25,00 \\
\hline Hepatomegalia & 15 & 13,90 \\
\hline Arañas vasculares & 12 & 11,10 \\
\hline
\end{tabular}

En relación a los hallazgos de laboratorio se encontró que las proteínas totales tuvieron un valor promedio de 6,06 gr/dl, y la albúmina, de 2,70 gr/dl. La creatinina tuvo una media de $1,54 \mathrm{mg} / \mathrm{dl}$ (Tabla 3). El valor promedio de sodio fue 136,00 $\mathrm{mEq} /$; y el del colesterol total, $159,60 \mathrm{mg} / \mathrm{dl}$. La fracción
HDL tuvo una media de 25,10 mg/dl, el colesterol LDL fue, en promedio, de $90,50 \mathrm{mg} / \mathrm{dl}$; y los triglicéridos mostraron valores promedio de $119,70 \mathrm{mg} / \mathrm{dl}$.

La media de hemoglobina fue 11,14 gr/dl, y el hematocrito 
fue $33,80 \%$. Los leucocitos tuvieron una media de 8424 . El promedio del tiempo de protrombina fue de 21,76 segundos; el índice internacional normalizado (INR de sus siglas en inglés) fue de 2,44 y las plaquetas fueron $137000 / \mathrm{mm}^{3}$.
La hipoalbuminemia se presentó en el $67,60 \%$ de los casos; la trombocitopenia, en el 58,30\%; anemia, en el 53,70\%; y la hipoprotrombinemia, en el 50,90\%.

Tabla 3. Valores de laboratorio

\begin{tabular}{|ccccccc|}
\hline Signos & N & Mínimo & Máximo & Media & Mediana & Desviación \\
\hline Bilirrubina total (mg/l) & 108 & 0,40 & 29,30 & 21,36 & 18,21 & 3,61 \\
\hline Albúmina (gr/dl) & 108 & 0,40 & 4,80 & 2,70 & 2,80 & 0,56 \\
\hline Tiempo de protrombina (segundos) & 108 & 10,70 & 60 & 21,76 & 17,30 & 2,43 \\
\hline Creatinina (mg/dl) & 108 & 0,60 & 19,00 & 1,54 & 0,80 & 0,58 \\
\hline INR & 108 & 0,98 & 13,20 & 2,44 & 2,23 & 0,52 \\
\hline Sodio (mEq/L) & 108 & 123 & 161 & 136 & 131 & 4,20 \\
\hline TGO (U/L) & 108 & 13 & 112 & 62,80 & 31,40 & 5,70 \\
TGP (U/L) & 108 & 9 & 157 & 41,30 & 27,70 & 5,30 \\
\hline Colesterol total (mg/dl) & 108 & 67 & 219 & 159,60 & 148,30 & 6,30 \\
\hline
\end{tabular}

Respecto a las ecografías, el 41,60\% mostró la triada de micronódulos hepáticos, esplenomegalia y ascitis; en el $21,50 \%$ se encontró un patrón similar, pero sin esplenomegalia; y en el $14,30 \%$, se observaron los mismos hallazgos, pero sin ascitis (Tabla 4).

Tabla 4. Hallazgos ecográficos

\begin{tabular}{|lcc|}
\hline Ecografía abdominal & Casos & $\%$ \\
\hline Ecografía abdominal normal & 1 & 1,20 \\
\hline Presencia de ascitis & 5 & 6,00 \\
\hline Hígado micronodular & 8 & 9,50 \\
\hline Esplenomegalia & 2 & 2,40 \\
\hline Hígado micronodular y ascitis & 18 & 21,40 \\
\hline Hígado micronodular y esplenomegalia & 12 & 14,30 \\
\hline Hígado micronodular, ascitis y esplenomegalia & 35 & 41,60 \\
\hline Ascitis y esplenomegalia & 3 & 3,60 \\
\hline Total & 84 & 100,00 \\
\hline
\end{tabular}


Se realizaron 11 biopsias hepáticas. Una de ellas reveló cirrosis biliar primaria, en 9 se observó cirrosis micronodular y una mostró combinación de lesiones micro y macronodulares.

La ascitis fue la complicación más común $(71,30 \%)$, la encefalopatía hepática se observó en el 47,20\%; seguida por la hemorragia por las varices esofágicas $(43,50 \%)$ (Tabla 5). Con respecto a las varices esofágicas, primaron las de segundo grado (20,40\%), seguidas de las de grado I $(13,00 \%)$ y grado III $(9,30 \%)$.

En relación a la encefalopatía hepática, la más frecuente fue de la de primer grado (19,50\%). El 14,70\% de los pacientes tuvieron encefalopatía de segundo grado, y solo el $13,10 \%$, de tercer grado. El síndrome hepatorrenal se presentó en el $8,30 \%$ de los casos, y la peritonitis bacteriana espontánea en el 5,60\%.

Tabla 5. Complicaciones de la cirrosis hepática

\begin{tabular}{|lcc|}
\hline Complicaciones & Número & $\%$ \\
\hline Ascitis & 61 & 56,50 \\
\hline Encefalopatía hepática & 51 & 47,20 \\
\hline Hemorragia digestiva alta & 39 & 36,10 \\
\hline Várices esofágicas & 47 & 43,50 \\
\hline Shock & 18 & 16,70 \\
\hline Infección bacteriana & 11 & 10,20 \\
\hline Síndrome hepatorrenal & 9 & 8,30 \\
\hline Insuficiencia hepática & 7 & 6,50 \\
\hline Peritonitis bacteriana espontánea & 6 & 5,60 \\
\hline
\end{tabular}

Puntaje de Child-Pugh: El puntaje de Child-Pugh más frecuente es el estadio B con un 25,90\% ( $n=28)$, luego el C con $13,90 \%(n=15)$ y, finalmente, el estadio A con $4,60 \%$ $(n=5)$. La estancia hospitalaria promedio fue 11,30 días, con un máximo de 90 días y un mínimo de 1 día. La mortalidad fue $33,30 \%(n=36)$ : las causas principales fueron el choque séptico y la insuficiencia hepática $(n=6)$, seguidos del choque hipovolémico y el síndrome hepatorrenal $(n=4)$. Se encontraron diferencias significativas entre los pacientes de sexo masculino y femenino: los varones presentaron con más frecuencia cirrosis alcohólica $(76,50 \%$ versus $42,50 \%$ en (as mujeres), ascitis, estadios avanzados de enfermedad y menor sobrevida $(58,80 \%$ en el sexo masculino versus $79,50 \%$ en el sexo femenino) $(p<0,05)$ (Tabla 6$)$.

Tabla 6. Diferencias en la presentación de la cirrosis, según género

\begin{tabular}{llcccccc}
\hline \multicolumn{1}{c}{ Género } & \multicolumn{2}{c}{ Masculino } & \multicolumn{2}{c}{ Femenino } \\
\hline Característica & $\mathrm{N} .^{\circ}$ & $\%$ & $\mathrm{~N} . .^{\circ}$ & $\%$ & Valor $p$ \\
\hline Alcoholismo & 52 & 76,50 & 17 & 42,50 & $0,005^{*}$ \\
\hline Ascitis & 34 & 85,00 & 43 & 63,20 & $0,012^{*}$ \\
\hline Estadio avanzado & 17 & 42,50 & 17 & 25,00 & $0,0048^{*}$ \\
\hline Sobrevida & 40 & 58,80 & 31 & 79,50 & $0,023^{*}$
\end{tabular}

$*: p<0,05$ 


\section{DISCUSIÓN}

En esta investigación, la edad promedio fue de 60,56 años, valor cercano al reportado por Bustíos ${ }^{(8)}$ en Lima, así como a los encontrado por Medina en Chile $\left(61,50\right.$ años) ${ }^{(2)}$. El estudio de Campollo, en Guadalajara, (1) reportó una población más joven, con un promedio de 55 años. El sexo masculino predominó $(62,90 \%)$, lo que es similar a lo hallado en el Hospital Rebagliati $(54,90 \%){ }^{(8)}$, Chile $(60,8 \%){ }^{(2)}$ y Guadalajara $(69,40 \%)^{(1)}$.

En relación a la etiología, en el 63 \% de los pacientes, el alcohol fue la causa; este resultado es superior al de Lima $(28,00 \%)^{(7)}$ y de Lebroc $(33,30 \%){ }^{(9)}$, pero similar al del Hospital Juárez $(57,4 \%){ }^{(10)}$ y al de Lambayeque, el cual obtuvo un odds ratio de 16 para esta etiología (11), aunque inferior al de Guadalajara $(77,7 \%)^{(1)}$.

Según DEVIDA ${ }^{(12)}$, el consumo de alcohol en Lima sigue aumentando, los peruanos empiezan a ingerir bebidas alcohólicas, en promedio, a los 13 años la edad. Según datos de la misma institución, el 59,70\% de los adolescentes de 12 a 18 años consume alcohol. El 34,00 \% de los consumidores jóvenes peruanos tienen un patrón patológico de consumo de alcohol. Carecemos de datos en Huancayo, pero la ingesta podría ser similar o superior.

Como segunda causa de cirrosis se halló a la hepatitis B $(7,40 \%)$, este valor es inferior al reportado en un estudio en el Hospital Rebagliati, que fue de 15,20\%. El Perú tiene áreas geográficas con alta endemicidad para infección por virus de hepatitis B como Madre de Dios, Abancay, Huanta y la Amazonía en general; en dichas áreas la prevalencia de seropositividad para el antígeno de superficie sería de $10-20 \%{ }^{(13)}$.

Un $25,00 \%$ de los casos fue catalogado como cirrosis criptogénica, este resultado es mayor al del Hospital Rebagliati donde esta enfermedad se presentó en el $21,30 \%$ (7). La hepatitis C se observó solo en el 2,78 \%, a diferencia del Hospital Rebagliati en el cual se halló en el $11,80 \%$. No se describieron casos de hepatitis autoinmune y se reportó un individuo con cirrosis biliar primaria.

La distensión abdominal (81,30 \%) fue el hallazgo más frecuente en el examen físico, seguido por la matidez desplazable $(75,00 \%)$ y la ascitis $(71,30 \%)$, mientras que Lebroc (Cuba) $\left.{ }^{9}\right)$ reportó como signo más frecuente a la ictericia $(47,90 \%)$, y la ascitis solo en el $39,50 \%$.

La prevalencia de anemia fue de 53,70\%, proporción inferior al promedio $(75,00 \%){ }^{(14,15)}$ y al valor hallado por Mathurin (Argentina) ${ }^{(16)}(86,00 \%)$, lo cual se debería a la mayor producción de eritropoyetina en la altura ${ }^{(7)}$, aunque se desconoce si podría cambiar el pronóstico del paciente cirrótico.
El cuadro clínico silencioso, con síntomas inespecíficos como fatiga e hiporexia, se presentaría en 14 al $40 \%$ de los cirróticos ${ }^{(14)}$. En el presente estudio primaron las manifestaciones de hipertensión portal, como la ascitis y la esplenomegalia. Respecto a los estigmas hepáticos, se encontraron pocos casos de eritema palmar y presencia de arañas vasculares (11\%).

El valor promedio de albúmina fue de $2,70 \mathrm{gr} / \mathrm{dl}$, al igual que en la serie de Lima ${ }^{(8)}$, pero con un valor mínimo de $0,40 \mathrm{mg} / \mathrm{dl}$ en un paciente. La media del tiempo de protombina fue de 21,70 segundos, con un valor máximo de un minuto.

El promedio de plaquetas fue de 137000 por campo; y se observa que más de la mitad de los pacientes presentaba trombocitopenia. La concentración media de bilirrubina fue de $4,25 \mathrm{mg} / \mathrm{dl}$ mientras que en un estudio previo ${ }^{(8)}$, fue de $4,65 \mathrm{mg} / \mathrm{dl}$.

El valor promedio de sodio fue de $136 \mathrm{meq} / \mathrm{L}$, resultado similar al de la serie de Lima ${ }^{(8)}$, en la cual fue de $135 \mathrm{meq} / \mathrm{L}$, con una tasa de $25 \%$ de hiponatremia en los pacientes con ascitis. Los pacientes con cirrosis desarrollan hiponatremia por disminución del líquido extracelular (hiponatremia hipovolémica) o por retención de sodio renal con sobrecarga de volumen (hiponatremia hipervolémica o dilucional).

En el Hospital Rebagliati, los pacientes cirróticos eran hospitalizados, principalmente, por sangrado de várices esofágicas $(38,00 \%)^{(8)}$, esta proporción es similar a la que encontramos en Huancayo.

La tasa de peritonitis bacteriana espontánea fue de 5,60\%, valor inferior a lo reportado previamente ${ }^{(8)}(5,60 \%$ versus $13,00 \%)$. Por el contrario, el $8,30 \%$ de los pacientes de Huancayo presentó síndrome hepatorenal mientras que en el estudio de Lima ${ }^{(8)}$ no se reportó ningún caso. Este hallazgo podría ser casual o estar asociado a una alteración de la función renal por efecto de la altura ${ }^{(17)}$.

En el estudio de Lebroc, en Cuba ${ }^{(9)}$, se encontró síndrome hepatorenal en el 1,65\%, de individuos, y el 52,00\% presentó sangrado digestivo alto.

No hubo pacientes cirróticos con hepatocarcinoma en la población estudiada, a diferencia de algunos casos en los estudios de Bustios ${ }^{\left({ }^{8}\right)}$ y Lebroc ${ }^{(9)}$. Esto se debería al exiguo porcentaje de pacientes con hepatitis $B$ que se observó en Huancayo.

La hemorragia digestiva por várices esofágicas fue de $43,500 \%$, un porcentaje muy similar al de Lima $(38,00 \%){ }^{(8)}$, Cuba ${ }^{(9)}(52,00 \%)$ y Guadalajara $(48,40 \%)^{(1)}$.

El 47,20\% de los pacientes presentó encefalopatía hepática, proporción superior a la hallada en Juárez $(29,00 \%){ }^{(10)}$ y 
Guadalajara $(24,80 \%)^{(1)}$.

El estadio de Child-Pugh más frecuente fue el $B$ con un $58,30 \%$, similar al de Cuba ${ }^{(9)}(44,00 \%){ }^{(10)}$, a diferencia de Lima ${ }^{(8)}$, donde predominó el estadio C $(42,50 \%)$.

La población estudiada presentó complicaciones asociadas, principalmente, a la hipertensión portal y, con menos frecuencia, a la insuficiencia hepática. No se puede discernir si esta evolución está relacionada con la altura y el ambiente hipóxico o con las características sociodemográficas de la población.

Los pacientes cirróticos presentan hipoxia crónica en los sinusoides hepáticos ${ }^{(18)}$, lo que induce a mayor producción de factor inducido por la hipoxia 1 (HIF-1, de sus siglas en inglés) y óxido nítrico, lo que conduce a una mayor vasodilatación de la arteria hepática. Esta elevación del HIF-1 es un componente normal de la adaptación a la altura (7) y se asocia a mayor eritropoyesis, hipertensión pulmonar e hipertensión en el ventrículo derecho, este último componente se incrementa en paralelo a la severidad del daño hepático ${ }^{(19)}$. Estos datos nos indican que el paciente cirrótico en la altura tendría mayores niveles de HIF-1, óxido nítrico y presión ventricular derecha, pero queda por definir las consecuencias de estos cambios fisiopatológicos. A pesar de lo expuesto, en modelos animales se ha demostrado que la hipoxia no se asociaría a mayor mortalidad en el paciente cirrótico ${ }^{(20)}$.

En el Perú y en el ámbito mundial no existen estudios sobre cirrosis en la altura ni acerca de la función hepática en la altura. Berendsohn en el año $1962{ }^{(21)}$ realizó un estudio comparativo en sujetos nativos y residentes de altura (4545 m s. n. m.) con un grupo control a nivel del mar, encontró elevada la bilirrubina total en los sujetos de altura (un aumento de bilirrubina indirecta más que directa). Estos resultados no necesariamente indicaban disfunción hepática y estaban relacionados con el aumento de hemoglobina y la producción de eritrocitos en la altura. Por otro lado, las globulinas séricas, fosforo inorgánico y la fosfatasa alcalina estaban incrementados, pero las proteínas séricas y las transaminasas fueron normales en ambos grupos.

Jurgens et al. (22) evaluaron a 12 sujetos sanos que vivían al nivel del mar y que fueron expuestos a hipoxia inducida por la altitud durante 7 días a $4559 \mathrm{~m}$. Se observaron pequeños cambios en la actividad de los citocromos CYP2D6 y CYP3A4 que sugieren que la hipoxia aguda no tiene efectos clínicamente significativos sobre los citocromos hepáticos en humanos.

Otro estudio ${ }^{(23)}$ examinó el efecto de la hipoxia aguda en los citocromos: CYP1A2 (metabolismo de teofilina) y CYP3A4 (verapamilo) en 20 sujetos en una cámara hipóxica normobárica. La hipoxia aguda no alteró la farmacocinética de ninguno de los dos fármacos ni de sus metabolitos principales. Por otro lado, Kalson ${ }^{(24)}$ investigó el flujo sanguíneo venoso portal, evaluado por Doppler, en un ambiente con hipoxia hipobárica, sin encontrar alteraciones significativas. Sin embargo, hasta la fecha no se habían descrito las características clínicas de la cirrosis en la altura.

El presente estudio cuenta con las siguientes limitaciones: al ser retrospectivo, no ha podido evaluar todas las características clínicas y complicaciones usualmente descritas en la cirrosis. Como se realizó en una sola localidad, no se pudo comparar las variaciones de la enfermedad a diferentes altitudes. Por último, al carecer de biopsia hepática en la mayoría de los casos, un grupo de enfermedades permaneció sin causa identificable y fueron catalogadas como cirrosis criptogénica.

Se concluye que la cirrosis hepática en la ciudad de Huancayo tiene como etiología principal la ingesta alcohólica, con bajas tasas de hepatitis B y C. Las complicaciones principales fueron la ascitis, la encefalopatía hepática y la hemorragia digestiva alta. El sexo masculino se asoció a mayor mortalidad, presencia de ascitis y estadios más avanzados de la clasificación de Child-Pugh. A diferencia de los estudios en poblaciones a nivel del mar, los pacientes cirróticos en esta población de altura tuvieron menores tasas de anemia, hepatocarcinoma y peritonitis bacteriana espontánea, aunque presentaron una mayor proporción de síndrome hepatorrenal y encefalopatía hepática. Se requieren estudios prospectivos que evalúen las repercusiones fisiopatológicas de la altura en el paciente cirrótico.

Contribuciones de los autores: W. Calderón concibió la idea. W Calderón, M Ascanio y P Yarinsueca elaboraron el primer borrador del manuscrito, revisaron la versión final, realizaron la recolección y análisis de los datos, así como la búsqueda bibliográfica; revisaron el borrador y elaboraron la versión final.

Fuente de financiamiento: Este artículo ha sido financiado por los autores.

Conflicto de interés: Los autores declaran no tener ningún conflicto de interés.

\section{REFERENCIAS BIBLIOGRÁFICAS}

1. Campollo O, Valencia-Salinas JJ, Berumen-Arellano A, PérezAranda MA, Panduro-Cerda A, Segura-Ortega J. Características epidemiológicas de la cirrosis hepática en el Hospital Civil de Guadalajara. Salud Pública Mex. 1997; 39(3): 195-200.

2. Medina E, Kaempffer A. Cirrosis hepática en Chile. Rev Chil Salud Pública. 2002; 6 (1): 3.

3. Farfán G, Cabezas C. Mortalidad por enfermedades digestivas y 
hepatobiliares en el Perú, 1995-2000. Rev Gastroenterol Perú. 2002; 22(4): 212-33.

4. Biblioteca Virtual en Salud. Análisis de la Situación de Salud Junín-2006. Oficina de Epidemiología de la DIRESA Junín [internet]. 2018. Disponible en: http://www.bvsde.paho. org/documentosdigitales/bvsde/texcom/asisregiones / Junin/ Junin2006.pdf

5. Dávalos Moscol M. Epidemiología de la cirrosis hepática en el Perú. Red Peruana de Bibliotecas en Salud. 2003; 6(2): 26-7.

6. Hamad N, Travis S. Gastrointestinal function. In: Swenson E, Bärtsch P, eds. High Altitude: Springer: New York; 2014.

7. Lumbreras L, León-Velarde F. El medio ambiente en Los Andes. En: Monge $C$, León-Velarde $F$, eds. El reto fisiológico de vivir en Los Andes. Lima: IFEA-UPCH; 2003. pp. 29-38.

8. Bustíos C, Dávalos M, Román, R, Zumaeta E. Características epidemiológicas y clínicas de la cirrosis hepática en la Unidad de Hígado del HNERM EsSalud. Rev Gastroenterol Perú. 2007; 27(3): 238-45.

9. Lebroc PD, Reina AB, Camacho AJ, Massíp RM. Caracterización clínica de pacientes con cirrosis hepática en el Hospital Provincial Docente "Dr. Antonio Luaces Iraola". Mediciego. 2011; 17(2): 1-8.

10. Rodríguez-Magallán A, Valencia-Romero HS, Trinidad-Altamirano J. Etiología y complicaciones de la cirrosis hepática en el Hospital Juárez de México. Rev Hosp Jua Mex. 2008; 75(4): 247-56.

11. Beltrán-Jara A, Neciosup-Puican E. Factores de riesgo para cirrosis hepática en la población adulta de la Red Asistencial EsSalud Lambayeque. Rev Cuerpo Méd HNAAA. 2011; 4(2): 77-83.

12. Equipo de Redacción Perú 21. Consumo de alcohol aumenta entre las adolescentes peruanas. [internet]. 2016. Disponible en: https://peru21.pe/lima/consumo-alcohol-aumenta-adolescentesperuanas-236759. /

13. Ruiz E, Almonte M, Pizarro R, Celis J, Montalbeti JA, Urbano R. Infección con virus de la Hepatitis $B$ y Hepatitis $C$ como factores de riesgo para hepatocarcinoma en el Perú: estudio de casos y controles. Rev Gastroenterol Perú. 1998; 18(3): 199-212.

14. Ginés $P$, Arroyo V. Cirrosis hepática. En: Farreras-Rozman, ed. Medicina interna. 17ma ed. Elsevier: Barcelona; 2014. pp. 250-3.

15. Mathurin SA, Agüero AP, Dascani NA, Prestera JA, Gianserra C, Londero $\mathrm{E}$, et al. Anemia en pacientes internados con cirrosis: prevalencia, significación clínica y factores predictivos. Acta Gastroenterol Latinoam. 2009; 39(2): 103-11.

16. González-Casas R, Jones EA, Moreno-Otero R. Spectrum of anemia associated with chronic liver disease. World J Gastroenterol. 2009; 15(37): 4653-8.

17. Hurtado A, Escudero E, Pando J, Sharma S, Johnson RJ. Cardiovascular and renal effects of chronic exposure to high altitude. Nephrol Dial Transplant. 2012; 27(4): 11-6.

18. Moeller M, Thonig A, Pohl S, Ripoll C, Zipprich A. Hepatic arterial vasodilation is independent of portal hypertension in early stages of cirrhosis. PLoS One. 2015; 10(3): e0121229.

19. Sepehrinezhad A, Dehghanian A, Rafati A, Ketabchi F. Impact of liver damage on blood-borne variables and pulmonary hemodynamic responses to hypoxia and hyperoxia in anesthetized rats. BMC Cardiovasc Disord. 2020; 20(1): 13.

20. Voiosu AM, Voiosu TA, Smarandache B, Radoi A, Mateescu RB, Baicus CR, et al. The impact of hypoxaemia on the outcome in liver cirrhosis. J Gastrointestin Liver Dis. 2016; 25(4): 481-7.

21. Berendsohn S. Hepatic function at high altitudes. Arch Intern Med. 1962; 109: 56-64.

22. Jurgens G, Christense HR, Brosen K, Sonne J, Loft S, Olven NV. Acute hypoxia and cytochrome p450-mediated hepatic drug metabolism in humans. Clin Pharmacol Ther. 2002; 71(4): 214-20.

23. Streit M, Goggelmann C, Dehnert C, Burhenne J, Riedel K, Menold
$\mathrm{E}$, et al. Cytochrome P450 enzyme-mediated drug metabolism at exposure to acute hypoxia (corresponding to an altitude of 4500m). Eur J Clin Pharmacol. 2005; 6(1): 39-46.

24. Kalson NS, Hext F, Davies AJ, Chan CW, Wright AD, Imray CH, et al. Do changes in gastro-intestinal blood flow explain hight-altitude anorexia?. Eur J Clin Invest. 2010; 40(8): 735-41.

\section{Correspondencia:}

Walter Calderón Gerstein

Teléfono: 981600344

Dirección: Jr. Mama Ocllo 2037, Lince. Lima, Perú.

Correo electrónico: waltercalderon2002@yahoo.com

Recibido: 02 de abril de 2020

Evaluado: 30 de abril de 2020

Aprobado: 16 de mayo de 2020

( ) La revista. Publicado por Universidad de San Martín de Porres, Perú (cc) BY Licencia de Creative Commons Artículo en acceso abierto bajo términos de Licencia Creative Commons Atribución 4.0 Internacional. (http://creativecommons.org/licenses/by/4.0/)

ORCID iDs

Walter Calderón Gerstein

Ascanio Paredes, Maritza

(D) https://orcid.org/0000-0002-5187-7705

Yarinsueca Mata, Pilar Rocí

https://orcid.org/0000-0002-1762-5088

(]) https://orcid.org/0000-0003-2323-4902 